\title{
DigiTraln 4.0: Ein Beratungskonzept für die Transformation in eine digitale Arbeitswelt
}

\author{
Stephan Kaiser (D), Arjan Kozica, Bianca Littig, Madlen Müller, \\ Ricarda Rauch und Daniel Thiemann
}

\section{Zielsetzung und Vorgehen}

Digitale Technologien und Innovationen verändern die Arbeitswelt in starkem Maße. Zukunftsfähige Unternehmen sind durch digitale Kommunikation und Vernetzung geprägt. Mitarbeitende und Führungskräfte entscheiden zunehmend auf Basis der Analyse großer Datenbestände. Institutionelles und individuelle Wissen wird in digitalen Informationsräumen geteilt und mobile Technologien erleichtern mehr und mehr ortsunabhängiges, flexibles Arbeiten. Basierend auf diesen vielfältigen Entwicklungen fragen sich viele Entscheider derzeit, was die digitale Transformation der Arbeitswelt für sie konkret bedeutet und wie sie diese im eigenen Unternehmen aktiv gestalten können.

Die Antworten auf diese Fragen werden durch die Komplexität der Transformation von Arbeitswelten erschwert [4]. Die Komplexität ergibt sich aus der Multidimensionalität der digitalen Transformation der Arbeitswelt. Vielfältige Aspekte wie die betroffenen Akteure, deren Arbeitsumfeld samt Strukturen, Abläufen, Ausstattung, aber auch konkretes Verhalten in den Bereichen Kommunikation und Führung sowie unternehmenskulturelle Aspekte sind hier zu nennen. Wissenschaftlich fundierte Erkenntnisse

Autoren gelistet in alphabetischer Reihenfolge.

S. Kaiser $(\square) \cdot$ B. Littig $\cdot$ R. Rauch

Universität der Bundeswehr München, Professur für Personalmanagement und Organisation, Neubiberg, Deutschland

A. Kozica · M. Müller · D. Thiemann

Hochschule Reutlingen, Professur Organisation und Leadership, Reutlingen,

Deutschland 
darüber, wie einzelne Dimensionen der Arbeitswelt von der digitalen Transformation beeinflusst werden, können diese Komplexität für Unternehmen in einem ersten Schritt verständlich und greifbar machen. Zusätzlich benötigen Praktiker einen zielführenden Prozess und anwendungsorientierte Instrumente, um die Transformation in die digitale Arbeitswelt gestalten zu können.

Das vom Bundesministerium für Bildung und Forschung (BMBF) und dem Sozialfonds der Europäischen Union im Rahmen des Programms Zukunft der Arbeit geförderte Projekt DigiTraIn 4.0 adressiert diese Herausforderungen. Auf Basis eines systematischen und wissenschaftlich fundierten Vorgehens entwickelten, erprobten und evaluierten die Mitarbeitenden des Projekts vier anwendungsorientierte Instrumente, mit denen Unternehmen die Transformation in die digitale Arbeitswelt erfolgreich gestalten können:

- Der Digitalisierungsatlas stellt als Bezugsrahmen für Unternehmen die wichtigsten, sich durch die digitale Transformation verändernden Dimensionen strukturiert vor und verweist auf Zusammenhänge zwischen den Dimensionen.

- Mit dem Digitalisierungsindex können Unternehmen den momentanen Ist-Zustand ihrer Arbeitswelt bezüglich aller Dimensionen der Digitalisierung ermitteln.

- Als drittes Instrument ermöglicht der Digitalisierungskompass Unternehmen, den für sie individuell sinnvollen Soll-Zustand festzulegen und zu erörtern, inwieweit eine Digitalisierung für bestimmte Dimensionen der Arbeitswelt angestrebt wird.

- Die Transformationsagenda bietet Unternehmen schließlich Orientierung darüber, wie sie den angestrebten Zielzustand durch aktives Veränderungsmanagement erreichen.

Im Folgenden werden die entwickelten Instrumente anhand von Anwendungsbeispielen näher beschrieben sowie ein Ausblick auf die weitere Forschungsarbeit gegeben.

\subsection{DigiTraln 4.0: Beratungsprozess und integrierte Instrumente}

\subsubsection{Ein Beratungskonzept zur Transformation in die Arbeit 4.0}

Übergeordnetes Ziel des Beratungsprozesses von DigiTraIn 4.0 ist, Unternehmen darin $\mathrm{zu}$ befähigen, ihre Arbeitswelt eigenständig und nachhaltig auf die Möglichkeiten und Herausforderungen der Digitalisierung ausrichten zu können. Das vierstufige Beratungskonzept orientiert sich an prozessualen Veränderungsmodellen des Change-Managements (z. B. [2]). Im ersten Schritt gilt es, die Entwicklungen durch die Digitalisierung und die daraus resultierende Veränderungsnotwendigkeit zu erkennen. Hieran schließt sich eine genauere Untersuchung des aktuellen Digitalisierungsgrades an, welche auf Veränderungsbedarfe in der Arbeitswelt hinweist. Im dritten Schritt 
werden Veränderungsmaßnahmen entwickelt, deren Implementierung im letzten Schritt vorbereitet wird.

Die anwendungsorientierten Instrumente des Projekts DigiTraIn 4.0 begleiten Unternehmen durch diese vier Phasen. Im Beratungsverständnis nimmt das Projekt die Grundidee der Prozessberatung [6] auf. Hierbei ist und bleibt der Klient (die Mitarbeitenden eines Unternehmens) der Experte im Unternehmen und entscheidet somit selbst darüber, wie er seine Arbeitswelt inhaltlich ausgestalten möchte. Der Berater dahingegen ist der Experte im Prozess zur Erarbeitung der digitalen Arbeitswelt. Er unterstützt den Klienten darin, Veränderungspotenziale und -risiken wahrzunehmen, zu verstehen und wirksame Handlungsmaßnahmen herzuleiten. Hierfür vereint das Beratungskonzept unterschiedliche Methoden, die interaktiv (z. B. in einem Workshop) bearbeitet werden können. Nachfolgend werden die vier Beratungsschritte von DigiTraIn 4.0 und die darin angewendeten Instrumente vorgestellt.

\subsubsection{Die Notwendigkeit zur Veränderung erkennen mit dem Digitalisierungsatlas}

Der Digitalisierungsatlas ist das erste Instrument im DigiTraIn 4.0-Beratungsprozess. Er dient dazu, die Veränderungen in der Arbeitswelt der Unternehmen, die durch die Digitalisierung entstehen, greifbar und beschreibbar zu machen. Somit stellt er einen Bezugsrahmen dar, um die Veränderungen der Arbeitswelt zu strukturieren und relevante Zusammenhänge zu erkennen. Dadurch bietet er den Unternehmen Orientierung für den Veränderungsprozess.

Erarbeitet wurde der Digitalisierungsatlas auf Basis einer systematischen Literaturanalyse sowie eigener empirischer Analysen. Im Rahmen dessen wurden zudem die Risiken und Chancen der digitalen Arbeitswelt analysiert [3]. Dabei wurden zehn relevante Dimensionen identifiziert, in denen Veränderungen der Arbeitswelt stattfinden, und in weitere Subdimensionen und Aspekte aufgeteilt (vgl. [7]). Diese zehn Dimensionen lassen sich auf drei Ebenen, Organisation, Interaktion und Individuum, aggregieren. Auf der organisationalen Ebene finden sich Dimensionen der Arbeitswelt, die das gesamte Unternehmen betreffen. Auf der individuellen Ebene liegt der Fokus dagegen auf Dimensionen, die (primär) den einzelnen Mitarbeitenden betreffen. Die Dimensionen auf der interaktionalen Ebene wiederum betreffen den Austausch und die Zusammenarbeit zwischen den Mitarbeitenden eines Unternehmens. So spannt der Atlas ein vollständiges Bild der Veränderungen in der Arbeitswelt der Unternehmen auf.

Der Fokus auf der organisationalen Ebene liegt auf vier Dimensionen, die dem Arbeitskontext im Unternehmen zuzurechnen sind. Diese Dimensionen umfassen neben Strukturen und Prozessen auch die digitale Infrastruktur und Koordination der Arbeit sowie die Unternehmensstrategie und -kultur. So wird beispielsweise die Spezifikation der Aufbau- und Ablauforganisation in einer digitalen Arbeitswelt ebenso betrachtet wie die strategische und die kulturelle Ausrichtung des Unternehmens auf eine digitale 
Arbeitswelt. Außerdem gehören beispielsweise die Ausgestaltung und Nutzung der digitalen Infrastruktur sowie datengestützte Entscheidungsprozesse und Kontrolle von Arbeit zur organisationalen Ebene des Digitalisierungsatlas.

Auf der interaktionalen Ebene können die Dimensionen Führung und Zusammenarbeit angesiedelt werden. Dabei umfasst die Dimension der Zusammenarbeit nicht nur die Subdimensionen interne und externe Zusammenarbeit, sondern auch die Kommunikation über digitale Tools sowie den Bereich der Mensch-Maschine-Interaktion. In der Dimension der Führung werden sowohl die operative Führung und Zusammenarbeit zwischen Mitarbeitenden und Führungskräften als auch die strategische Führung des Top-Managements betrachtet.

Die individuelle Ebene umfasst schließlich alle Dimensionen der Arbeitswelt, die sich primär auf die Mitarbeitenden als Individuen beziehen. Dazu gehören neben den Kompetenzen der Mitarbeitenden in der digitalen Arbeitswelt auch veränderte Arbeitsaufgaben und Rollen. Zudem sind die Motivation für diese Veränderungen im Rahmen der digitalen Transformation sowie die physische und psychische Gesundheit der Mitarbeitenden wichtige Dimensionen auf der individuellen Ebene.

Dabei sind die Ebenen und Dimensionen des Digitalisierungsatlas nicht als separate Elemente zu verstehen, sondern es bestehen vielfältige Wechselwirkungen zwischen den Ebenen und den einzelnen Dimensionen. Hier dient der konfigurationstheoretische Ansatz [5] als theoretische Grundlage, aus dessen Perspektive die unterschiedlichen Dimensionen von Unternehmen integrativ berücksichtigt sowie Wechselwirkungen explizit betrachtet werden können.

Der Digitalisierungsatlas bietet somit Entscheidern in Unternehmen Orientierungshilfen, um die digitale Transformation der Arbeitswelt zielgerichtet vorantreiben zu können. Dabei liefert er Antworten auf die grundlegende Frage „Welche Dimensionen der Arbeitswelt betrifft die Digitalisierung und welche Wechselwirkungen bestehen zwischen den Dimensionen?“" und stellt somit die Ausgangsbasis und konzeptionelle Grundlage der weiteren DigiTraIn 4.0-Instrumente dar.

\subsubsection{Veränderungsbedarfe und -potenziale ableiten mit dem Digitalisierungsindex}

Der Digitalisierungsindex hat zum Ziel, den digitalen Reifegrad einer Arbeitswelt zu erfassen. Er unterstützt Unternehmen dabei, sich in der Digitalisierung zu verorten und relevante Veränderungsbedarfe zu identifizieren. Der Digitalisierungsindex formt somit eine belastbare Basis für die Entwicklung konkreter Veränderungsmaßnahmen im Digitalisierungskompass.

Das Instrument besteht aus einem quantitativen Fragebogen, der die organisationalen, interaktionalen und individuellen Dimensionen des Digitalisierungsatlas misst. Der Index wurde in einer Lang- und Kurzversion entwickelt. 
Die Langversion des Digitalisierungsindex umfasst einen Multi-Item-Fragebogen mit 88 Fragen und wurde für die Anwendung im Rahmen des Beratungskonzeptes entworfen. Hierbei wird beabsichtigt, möglichst viele Beschäftigte eines Unternehmens aus administrativen und kreativ-dispositiven Tätigkeitsbereichen einzubeziehen. Die Fragen sind so konzipiert, dass sie die Einschätzungen der Mitarbeitenden zu einzelnen Aspekten ihrer Arbeitswelt erfragen. Das Ergebnis umfasst dann einen digitalen Reifegrad je Arbeitsweltdimension. Somit kann ein Unternehmen zum einen erkennen, wie stark die Arbeitswelt insgesamt bereits digital ausgerichtet ist, zum anderen wird aufgeschlüsselt, welche Dimensionen noch Entwicklungspotenzial aufweisen.

In einem Anwendungsbeispiel wurde die Langversion des Digitalisierungsindex in einem Unternehmen der Gesundheitsbranche durchgeführt. Hierbei erhielten insgesamt 2.702 Mitarbeitende eine Einladung per Mail, den Digitalisierungsindex online auszufüllen. Die mittlere Bearbeitungszeit lag bei 22 min. Über einen Befragungszeitraum von vier Wochen nahmen 810 Personen an der Befragung teil.

Der Digitalisierungsindex stellte sich als ein geeignetes Instrument dar, um die im Digitalisierungsatlas entwickelten Dimensionen, Subdimensionen und Aspekte zu indizieren bzw. messbar zu machen. Abb. 27.1 zeigt anhand fiktiver Zahlen für die Dimension „Digitale Infrastruktur“ auf der organisationalen Ebene die Mittelwerte für die einzelnen Subdimensionen und Aspekte auf. Die dargestellten Mittelwerte können Werte von 1 bis 5 annehmen, wobei 1 auf einen sehr geringen und 5 einen äußerst hohen Digitalisierungsgrad hinweist.

Bei dem untersuchten Unternehmen aus der Gesundheitsbranche offenbarten die Index-Werte ein gemischtes Bild. So war insgesamt ein überdurchschnittlich hoher Digitalisierungsgrad zu verzeichnen, jedoch zeigten einige Indizes der Aspekte auf deutlichen Entwicklungsbedarf hin. Hierzu gehörte beispielsweise der Aspekt der physischen Gesundheit von Mitarbeitenden. Ebenso wurde festgestellt, dass Mitarbeitende die digitale Reife des Unternehmens auf organisationaler Ebene besser bewerteten als die Führungskräfte. Auf individueller Ebene war es genau umgekehrt: Hier bewerten die Führungskräfte die digitale Reife höher als die Mitarbeitenden.

Die Kurzversion des Digitalisierungsatlas wurde entwickelt, um Personen eine erste Einschätzung über den Digitalisierungsgrad ihrer Arbeitswelt zu geben. Er ist online innerhalb von fünf Minuten durchführbar und präsentiert dem Teilnehmenden am Ende eine automatisierte Ergebnisseite über die Reifegrade seiner digitalen Arbeitswelt.

Der Kurzindex wurde branchenübergreifend bei 23 überwiegend kleinen und mittleren Unternehmen durchgeführt. Diese Umfrage zeigte, dass der Großteil dieser Unternehmen die Arbeitswelt bereits mittelmäßig bis sehr digital ausrichtet. Die Gesamtbetrachtung der Reifegrade zeigte, dass die digitale Infrastruktur, Strukturen, Prozesse, Kultur, Zusammenarbeit und Kommunikation, Führung und Gesundheit in diesen Unternehmen bereits mittelmäßig bis stark digital ausgerichtet waren. Die größten Entwicklungspotenziale wiesen diese Unternehmen in den Dimensionen Motivation, Arbeitsaufgaben und -rollen, Kompetenzen, Strategie und Koordination der Arbeit auf, welche zum damaligen Zeitpunkt wenig bis mittelmäßig digital ausgerichtet waren. 


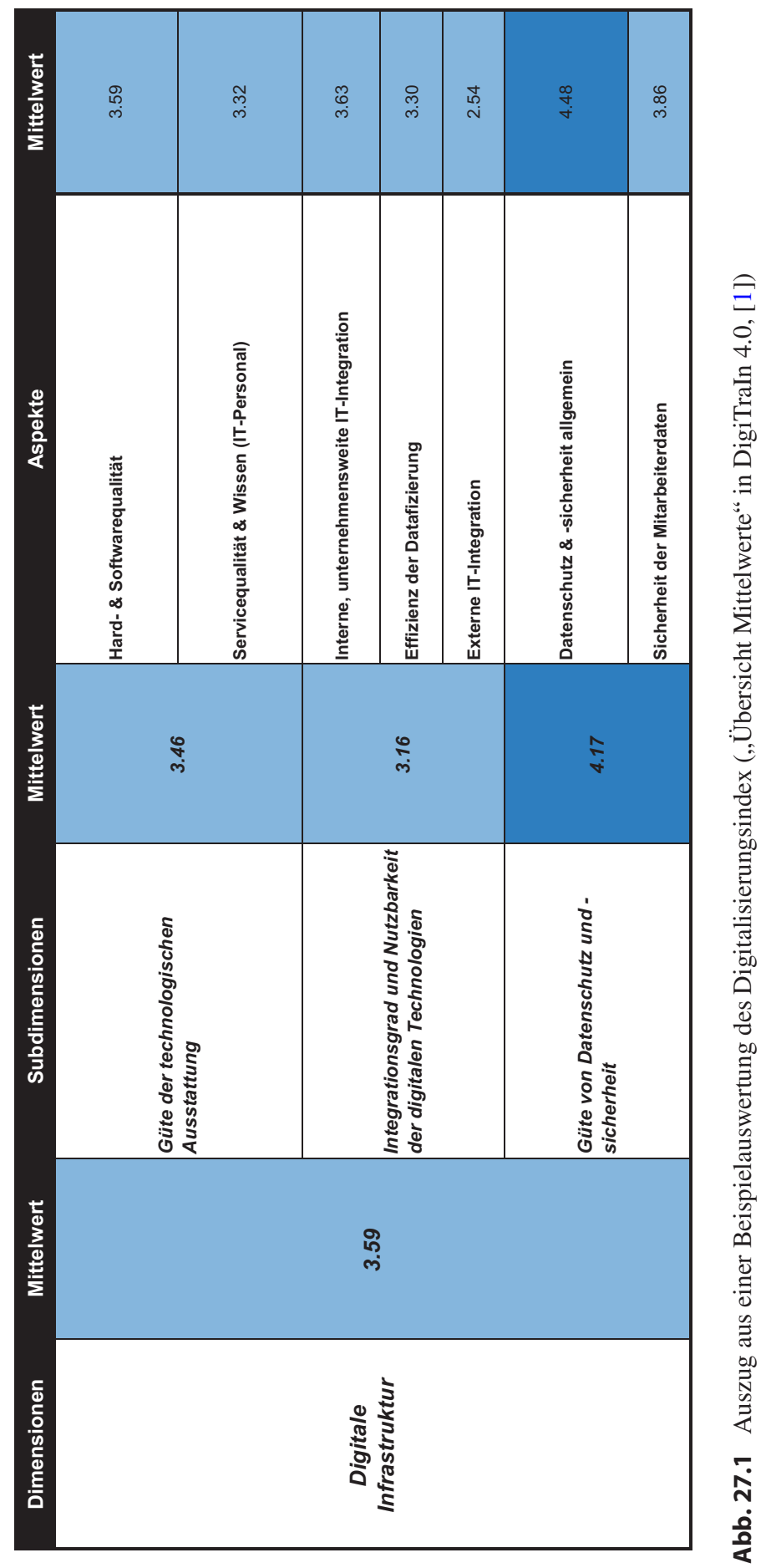




\subsubsection{Eine stimmige digitale Arbeitswelt planen mit dem Digitalisierungskompass}

Über die Kenntnis des Status quo hinaus, ist es für Entscheider von hoher Bedeutung, eine Vorstellung des angestrebten Zielzustands der unternehmensspezifischen digitalen Arbeitswelt zu haben. Daher unterstützt der Digitalisierungskompass Unternehmen dabei, ihre Zielgrößen der digitalen Transformation der Arbeitswelt festzulegen. Das Instrument stellt ein auf wissenschaftlicher und empirischer Basis konzipiertes Workshopformat dar. Es ist vor allem für kleine und mittlere Unternehmen geeignet, die in ihrem Unternehmen Digitalisierungsmaßnahmen der Arbeitswelt strategisch planen und umsetzen wollen. Das Workshopformat beinhaltet verschiedene Module, die je nach Bedarf von den Unternehmen bearbeitet werden können. Die wichtigsten Themenfelder des Workshops sind:

1. Identifikation von relevanten internen und externen Faktoren, die die Digitalisierung der Arbeitswelt betreffen: Das erste Modul basiert auf dem Digitalisierungsatlas und ermöglicht es Unternehmen, ein umfassendes Verständnis über verschiedene Dimensionen der digitalen Arbeitswelt und deren Zusammenhänge zu gewinnen. Dazu wird ein eigens entwickeltes Brettspiel eingesetzt, das die Dimensionen des Digitalisierungsatlas und deren Zusammenhänge auf interaktive Weise für Mitarbeitende und Führungskräfte greifbar macht. Als optionalen nächsten Schritt sieht der Digitalisierungskompass vor, unternehmensspezifische externe Faktoren zu identifizieren, die die digitale Transformation der Arbeitswelt beeinflussen. Im Fokus stehen dabei politische, wirtschaftliche, soziokulturelle, technologische, ökologischgeographische und rechtliche Einflussfaktoren.

2. Entwicklung einer Vision der digitalen Arbeitswelt: Mittels angeleiteter Brainstorming-Techniken und systematischer Moderation entwickeln die Workshopteilnehmenden die Grundzüge einer unternehmensspezifischen Vision der zukünftigen digitalen Arbeitswelt. Diese bildet die Basis, um firmenintern die Vision der Arbeitswelt genauer auszuarbeiten und eine visionäre Vorstellung über die Zukunft der Arbeit im Unternehmen zu entwickeln.

3. Bewertung des Ist-Zustandes im Unternehmen auf Basis der Index-Ergebnisse: In diesem Modul werden die unternehmensspezifischen Ergebnisse des Digitalisierungsindex aufgegriffen. Die Teilnehmenden stellen sowohl Stärken als auch Entwicklungspotenziale des Unternehmens anhand der Indexergebnisse dar und bewerten diese hinsichtlich ihrer Relevanz für die eigene Arbeitswelt.

4. Entwicklung eines konkreten Zielzustandes: Ausgehend von der zuvor entwickelten Vision werden im nächsten Modul unternehmensspezifische Digitalisierungsziele erarbeitet. Dies geschieht anhand vorgefertigter Leitfragen entlang der Dimensionen des Digitalisierungsatlas. Dadurch wird die Vision der Arbeitswelt auf konkrete Dimensionen bezogen und handhabbar gemacht.

5. Identifikation von unternehmensspezifischen Handlungsbedarfen und kritischen Variablen: Die definierten Zielzustände für die einzelnen Dimensionen werden mit 
dem Status quo des Unternehmens abgeglichen. Durch den Soll-Ist-Vergleich kann konkret ermittelt werden, in welchen Dimensionen Handlungsbedarf besteht und wie diese zu priorisieren sind. Anschließend werden die Wechselwirkungen zwischen den einzelnen Dimensionen genauer betrachtet. Für die wichtigsten Dimensionen wird dabei ermittelt, wie stark sie die anderen Dimensionen beeinflussen und inwiefern sie selbst von diesen beeinflusst werden. Dadurch kann identifiziert werden, welche Dimensionen am einflussreichsten sind und erfolgskritische Treiber darstellen. Abschließend werden erste konkrete Handlungsbedarfe ausgearbeitet und priorisiert, um die aktive Transformationsphase vorzubereiten.

Als Teilnehmende des Workshops sollten diejenigen Personen einbezogen werden, deren Perspektiven für die Erarbeitung von Zielgrößen der digitalen Transformation der Arbeitswelt entscheidend sind (z. B. Führungskräfte, Mitarbeitende, Betriebsrat).

Zur Anwendung des Workshopformats benötigen die Unternehmen einen Moderator, der die einzelnen Module methodisch anleitet. Der Moderator kann sowohl extern als auch intern aus dem Unternehmen sein und sollte bereits erfahren darin sein, Workshops durchzuführen und zu gestalten. Der Moderator sollte den „Baukasten“ des Digitalisierungskompasses bei Bedarf methodisch und inhaltlich auf das jeweilige Unternehmen anpassen.

Der Digitalisierungskompass beansprucht in einer einfachen Ausführung einen halben bis ganzen Tag, abhängig davon, welche Module an einem Tag durchgeführt werden. Die einzelnen Elemente lassen sich aber zeitlich auch auseinanderziehen - je nach Diskussions- und Klärungsbedarf in den spezifischen Firmen.

Somit ermöglicht es der Digitalisierungskompass Unternehmen, wesentliche Ansatzpunkte systematisch zu identifizieren und zu priorisieren, ohne dabei das Gesamtbild aus den Augen zu verlieren. Auf Basis dessen können Unternehmen im folgenden Schritt mit der „Transformationsagenda“ in die Transformationsphase übergehen.

\subsubsection{Veränderungsmaßnahmen implementieren mit der Transformationsagenda}

Mithilfe der Transformationsagenda können Unternehmen konkrete Handlungsmaßnahmen für die im Digitalisierungskompass entwickelten Ziele planen. Um dies zu erreichen, unterstützt ein generisches Modell zunächst dabei, eine Change-Taktik zu entwickeln. Anschließend werden unternehmensspezifische Maßnahmen konkret in einen Handlungsplan überführt.

Um eine angemessene Change-Taktik zu entwickeln, arbeitet in einem ersten Schritt der/die Berater/in gemeinsam mit dem zu beratenden Unternehmen ein generisches Modell durch. Hierzu wurde im Projekt DigiTraIn 4.0 eine mehrdimensionale Matrix entwickelt, welche relevante Aspekte einer Change-Taktik umfasst. Für die Erarbeitung dieser Matrix wurden bisherige theoretische, konzeptionelle und empirische Studien 
recherchiert und analysiert. Mithilfe des generischen Modells der Transformationsagenda werden die Anwender durch vier Ebenen geführt:

1. Die organisationale Durchdringung (beispielsweise Reichweite und Umfang der Veränderung)

2. Den Modus (beispielsweise Dauer, Startpunkt und Ziel der Veränderung)

3. Die organisationale Einbindung (beispielsweise Antriebskräfte und Narrativ)

4. Den organisationalen Kontext (beispielsweise Erfahrungswissen im Bereich Digitalisierung und Veränderungsbereitschaft)

Im Vorfeld einer Transformation sollten Unternehmen die genannten Aspekte der Ebenen reflektieren und eine geeignete Ausprägung für das Unternehmen und Veränderungsvorhaben wählen. Die Change-Taktik setzt sich somit aus der individuellen Kombination der Ausprägungen zusammen und dient als Ausgangspunkt dafür, im nächsten Schritt Maßnahmen zu planen und durchzuführen.

Als zweiter Bestandteil der Transformationsagenda erarbeiten und planen die Unternehmen basierend auf den im Kompass identifizierten Zielen und Handlungsnotwendigkeiten anhand einer strukturierten Maßnahmentabelle ihre Handlungsschritte für die einzelnen Dimensionen. Hierbei werden sowohl der Zeitpunkt als auch die Dauer der in den jeweiligen Dimensionen stattfindenden Maßnahmen reflektiert und festgelegt. Weiterhin werden die Maßnahmen dadurch aufeinander abgestimmt und ihre Abhängigkeiten berücksichtigt.

Als Orientierungshilfe dienen den Teilnehmenden drei beispielhafte Transformationspfade für die digitale Arbeitswelt. Diese wurden durch das Projekt DigiTraIn 4.0 auf Basis einer Literaturrecherche und durch ein Experiment ermittelt. Inhaltlich wurden dazu drei Wandelanlässe ausgewählt, und zwar ein Wandelpfad für die Implementierung neuer Technologien, ein Wandelpfad zur Einführung flexiblen Arbeitens und ein Wandelpfad zur Ausrichtung auf eine Digitalstrategie im Unternehmen. Verschiedene Experten und Expertinnen haben zu diesen Wandelanlässen Lösungsprozesse entwickelt. Die Erkenntnisse aus dem Experiment wurden in zwei Fokusgruppen mit Führungskräften, Mitarbeitervertretungen sowie Change-Experten gespiegelt. Abschließend wurden die drei Wandelpfade beschrieben. In der Transformationsagenda gliedern sich die Transformationspfade in die wichtigsten Erkenntnisse zum jeweiligen Transformationspfad sowie die detaillierte Maßnahmen-Tabelle für die Dimensionen. Die Transformationspfade geben den Unternehmen somit anhand konkreter Beispiele Hilfestellung für die Entwicklung einer detaillierten Maßnahmenplanung für das eigene Transformationsvorhaben.

Mithilfe der unternehmensspezifischen Change-Taktik und der individuellen Maßnahmenplanung können Unternehmen nun durch die Transformationsagenda ihr Veränderungsvorhaben auf strukturierte Art und Weise umsetzen. Die Transformationsagenda rundet somit die Instrumente zur Unterstützung der digitalen Transformation der Arbeitswelt ab. 


\subsection{Ausblick}

In der letzten Phase des Projekts begleitet DigiTraIn 4.0 sechs Unternehmen auf ihrem Weg in die digitale Arbeitswelt. In dieser Phase durchlaufen die Unternehmenspartner schrittweise die im Projekt entwickelten Instrumente.

Im Anschluss an den Beratungsprozess werden die Instrumente und Maßnahmen evaluiert und auf dieser Basis weiterentwickelt. Außerdem entstehen im Zuge der Anwendung der Instrumente weitere Workshop-Leitfäden für die einzelnen Instrumente, die zusätzlich auf der DigiTraIn-Homepage veröffentlicht werden. So stehen interessierten Unternehmen, die ihre Arbeitswelt digitalisieren wollen, zahlreiche Hilfestellungen zur Anwendung der Instrumente zur Verfügung. Damit führt DigiTraIn 4.0 den bisher beschrittenen Weg fort, die wissenschaftlich fundierten Instrumente in die Unternehmensrealität zu bringen und für die Praxis (besonders für kleine und mittlere Unternehmen) anwendungsfreundlich zu gestalten.

Zudem werden die Beratungsprozesse bei den Unternehmenspartnern als Fallstudien dokumentiert und ausgearbeitet. So können interessante Erkenntnisse, wie beispielsweise typische Stolpersteine für Unternehmen, gesammelt und als Anwendungsbeispiele für andere Unternehmen veröffentlicht werden.

\section{Projektpartner und Aufgaben}

- Universität der Bundeswehr München

Analyse, Entwicklung und Erprobung von Instrumenten für die Transformation der organisationalen Dimension der Digitalisierung

- Hochschule Reutlingen Analyse, Entwicklung und Erprobung von Instrumenten für die Transformation der individuellen und interaktionalen Dimension der Digitalisierung

- AOK Baden-Württemberg

Entwicklung und Erprobung eines integrativen Best- Practice-Modells der Digitalisierung und der digitalen Transformation

- RKW Bayern e. V.

Analyse, Entwicklung und Erprobung von Beratungsansätzen für die KMUspezifische Digitalisierung

\section{Literatur}

1. DigiTraIn 4.0 (Hrsg.). (2020). Materialien Anwendungsleitfaden. Übersicht Mittelwerte. Zugriff am 08. Juni 2020. Verfügbar unter https://digitrain40.de/

2. Hayes J (2018) The theory and practice of change management, 5. Aufl. Palgrave Macmillan, Basingstoke, Hampshire 
3. Jager, A., Rauch, R. Thiemann, D., \& Kaiser, S. (2019). Die sechs Gefahren der digitalen Arbeitswelt - und was Sie dagegen tun können. Personalmagazin (Haufe), 01/2019, S 48-52

4. Kaiser S, Kozica A, Wittmann P (2017) Führung und Arbeit in einer digitalisierten und datengetriebenen Welt: Ein konfigurationstheoretischer Zugang. In: Krause S, Pellens B (Hrsg) Betriebswirtschaftliche Implikationen der digitalen Transformation. ZfbF-Sonderheft, vol 72/17. Springer Gabler, Wiesbaden, S 65-80

5. Meyer AD, Tsui AS, Hinings CR (1993) Configurational approaches to organizational analysis. Acad Manag J 36(6):1175-1195

6. Schein EH (1987) Process consultation (Addison-Wesley series on organization development, Reprinted with corrections). Addison-Wesley, Reading, Mass.

7. Thiemann D, Kozica A, Rauch R, Kaiser S (2019) Die Digitalisierung der Arbeitswelt verstehen und gestalten: Der Digitalisierungsatlas als mehrdimensionaler Bezugsrahmen. Zeitschrift für Führung und Organisation (ZfO). 02/2019, S 114-121

Open Access Dieses Kapitel wird unter der Creative Commons Namensnennung 4.0 International Lizenz (http://creativecommons.org/licenses/by/4.0/deed.de) veröffentlicht, welche die Nutzung, Vervielfältigung, Bearbeitung, Verbreitung und Wiedergabe in jeglichem Medium und Format erlaubt, sofern Sie den/die ursprünglichen Autor(en) und die Quelle ordnungsgemäß nennen, einen Link zur Creative Commons Lizenz beifügen und angeben, ob Änderungen vorgenommen wurden.

Die in diesem Kapitel enthaltenen Bilder und sonstiges Drittmaterial unterliegen ebenfalls der genannten Creative Commons Lizenz, sofern sich aus der Abbildungslegende nichts anderes ergibt. Sofern das betreffende Material nicht unter der genannten Creative Commons Lizenz steht und die betreffende Handlung nicht nach gesetzlichen Vorschriften erlaubt ist, ist für die oben aufgeführten Weiterverwendungen des Materials die Einwilligung des jeweiligen Rechteinhabers einzuholen.

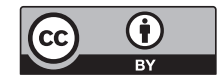

